\title{
A PROOF OF NEW SUMMATION FORMULAE BY USING SAMPLING THEOREMS
}

\author{
AHMED I. ZAYED
}

(Communicated by Andrew M. Bruckner)

\begin{abstract}
Using symbolic manipulation programs, William Gosper has obtained in the last two years new, but unusual, summation formulae involving trigonometric functions. Recently, Ismail and Zhang have been able to prove mathematically some of these formulae and generalize them to summation formulae involving the Bessel functions of the first kind.

In this paper we show that some of Gosper's formulae, as well as their generalization by Ismail and Zhang, can be obtained from already known results in sampling theory. Moreover, we show that sampling theory can actually produce other new summation formulae, involving different kinds of special functions, in a straightforward fashion.
\end{abstract}

\section{INTRODUCTION}

Using symbolic manipulation programs and a fair amount of ingenuity, William Gosper recently obtained new and unusual, summation formulae for series involving trigonometric functions. Some of these formulae have just been mathematically verified by Ismail and Zhang [7], who have also generalized some of these summation formulae to series involving the Bessel function of the first kind. Their proofs involve several special function relations together with some techniques borrowed from the theory of Fourier series and integrals.

As it turns out, some of these formulae can be easily obtained from already known results in sampling theory.

Although widely known and used in the fields of communication and information theory, sampling theory has only attracted moderate attention in the mathematical community, despite the fact that its roots can be traced back to the work of Cauchy; see [9].

The fundamental theorem in sampling theory, which is due to WhittakerShannon-Kotel'nikov, states that if $f$ is a $\sigma$-bandlimited function, i.e., for some $g \in L^{2}(-\sigma, \sigma)$

Received by the editors March 11, 1991 and, in revised form, June 21, 1991; presented at the ICIAM (91) International Conference on Industrial and Applied Mathematics, Washington, D.C., July 1991.

1991 Mathematics Subject Classification. Primary 40A30, 42C15; Secondary 33C20, 94A12.

Key words and phrases. Trigonometric series, Bessel functions, hypergeometric functions, Shannon and Kramer sampling theorems. 


$$
f(t)=\frac{1}{\sqrt{2 \pi}} \int_{-\sigma}^{\sigma} g(x) e^{i x t} d x
$$

then

$$
f(t)=\sum_{k=-\infty}^{\infty} f\left(t_{k}\right) S_{k}(t), \quad t \in R
$$

where

$$
S_{k}(t)=\frac{\sin \sigma\left(t-t_{k}\right)}{\sigma\left(t-t_{k}\right)}, \quad t_{k}=\frac{k \pi}{\sigma}
$$

are the sampling functions and sampling points respectively; see [1, 9] for more details.

This theorem has been extended to integral transforms of types other than the Fourier one by Kramer [10] and Weiss [12]. Although Weiss announced his result first, he never published his proof. This is why this generalization of the Whittaker-Shannon-Kotel'nikov sampling theorem is now known as Kramer's sampling theorem.

For convenience, we shall state Kramer's sampling theorem not only because it is not very well known, but also because it is widely used in the development of this paper. Kramer's theorem goes as follows:

Let $K(x, t)$ be in $L^{2}(I)$ as a function of $x$ for each real number $t$, where $I=[a, b]$ is some finite closed interval, and let $\mathbf{E}=\left\{t_{k}\right\}_{k \in \mathbf{Z}}$ be a countable set of real numbers such that $\left\{K\left(x, t_{k}\right)\right\}_{k \in Z}$ is a complete orthogonal family of functions in $L^{2}(I)$. If

$$
f(t)=\int_{a}^{b} g(x) K(x, t) d x
$$

for some $g$ in $L^{2}(I)$, then $f$ admits the sampling expansion

$$
f(t)=\sum_{k=-\infty}^{\infty} f\left(t_{k}\right) S_{k}^{*}(t)
$$

where

$$
S_{k}^{*}(t)=\int_{a}^{b} K(x, t) \bar{K}\left(x, t_{k}\right) d x / \int_{a}^{b}\left|K\left(x, t_{k}\right)\right|^{2} d x .
$$

When $K(x, t)=e^{i x t}, t_{k}=(\pi k / \sigma)$, and $[a, b]=[-\sigma, \sigma],(1.3)$ and (1.4) reduce to (1.1) and (1.2), respectively.

A prototype situation where the sampling points $\left\{t_{k}\right\}$ and sampling functions $S_{k}^{*}(t)$ arise, is when one solves certain types of selfadjoint boundary-value problems, usually regular Sturm-Liouville boundary-value problems.

In a series of papers $[15,16]$, we have shown the surprising fact that Kramer's sampling series $(1.4)-(1.5)$ is nothing more than a Lagrange-type interpolation series, provided that the kernel function $K(x, t)$ arises from either regular Sturm-Liouville boundary-value problems or some singular ones with discrete spectra. This result has also been shown to hold in higher dimensions [13], as well as for some boundary-value problems associated with $n$th order differential operators [14]. 
The aim of this paper is to show that some of these new summation formulae, due to Gosper, Ismail, and Zhang, can actually be obtained from already known results in sampling theory in an easy and straightforward fashion. In fact, we shall show that other summation formulae, which we believe to be new, can be obtained just as easily.

2. SUMMATION FORMULAE INVOLVING TRIGONOMETRIC AND BESSEL FUNCTIONS

We begin with

$$
\sum_{n=0}^{\infty} \frac{(-1)^{n} \sin \sqrt{b^{2}+\pi^{2}(n+1 / 2)^{2}}}{(n+1 / 2) \sqrt{b^{2}+\pi^{2}(n+1 / 2)^{2}}}=\frac{\pi}{2} \frac{\sin b}{b},
$$

which is due to Gosper, and its generalization

$$
\sum_{n=0}^{\infty} \frac{(-1)^{n} J_{\nu}\left(\sqrt{b^{2}+\pi^{2}(n+1 / 2)^{2}}\right)}{(n+1 / 2)\left[\sqrt{b^{2}+\pi^{2}(n+1 / 2)^{2}}\right]^{\nu}}=\frac{\pi}{2} b^{-\nu} J_{\nu}(b),
$$

which is due to Ismail and Zhang [7]; $J_{\nu}$ is the Bessel function of the first kind. Formula (2.2) is a generalization of (2.1) since the former reduces to the latter when $\nu=\frac{1}{2}$.

The following result can be obtained from Theorem 1 and Corollary 4 in [16] by a simple change of variables: if for some $g \in L^{2}(0, a)$

$$
f(t)=\int_{0}^{a} g(x) \cos (x t) d x
$$

then

$$
f(t)=\sum_{k=-\infty}^{\infty} f\left(\left(k+\frac{1}{2}\right) \frac{\pi}{a}\right) \frac{\sin (a t-(k+1 / 2) \pi)}{(a t-(k+1 / 2) \pi)},
$$

uniformly on compact subsets of $\mathbf{R}$. In particular,

$$
f(0)=\sum_{k=-\infty}^{\infty} f\left(\left(k+\frac{1}{2}\right) \frac{\pi}{a}\right) \frac{(-1)^{k}}{(k+1 / 2) \pi} .
$$

By putting $g(x)=\left(a^{2}-x^{2}\right)^{\nu / 2} J_{\nu}\left(b \sqrt{a^{2}-x^{2}}\right)$ in (2.3) and using formula (50) in $\S 1.13$ of [2], which states that

$$
\begin{aligned}
& \int_{0}^{a}\left(a^{2}-x^{2}\right)^{\nu / 2} J_{\nu}\left(b \sqrt{a^{2}-x^{2}}\right) \cos (t x) d x \\
& \quad=\sqrt{\frac{\pi}{2}} a^{\nu+1 / 2} b^{\nu} J_{\nu+1 / 2}\left(a \sqrt{b^{2}+t^{2}}\right)\left(b^{2}+t^{2}\right)^{-(\nu+1 / 2) / 2},
\end{aligned}
$$

for $\operatorname{Re} \nu>-1, b>0$, we obtain

$$
f(t)=\sqrt{\frac{\pi}{2}} a^{\nu+1 / 2} b^{\nu} J_{\nu+1 / 2}\left(a \sqrt{b^{2}+t^{2}}\right)\left(b^{2}+t^{2}\right)^{-(\nu+1 / 2) / 2} .
$$

Hence, (2.4) becomes

$$
\begin{aligned}
\frac{J_{\nu+1 / 2}\left(a \sqrt{b^{2}+t^{2}}\right)}{\left[b^{2}+t^{2}\right]^{(\nu+1 / 2) / 2}}=\sum_{k=-\infty}^{\infty} \frac{J_{\nu+1 / 2}\left(\sqrt{a^{2} b^{2}+(k+1 / 2)^{2} \pi^{2}}\right)}{\left[b^{2}+(k+1 / 2)^{2} \pi^{2} / a^{2}\right]^{(\nu+1 / 2) / 2}} \\
\times \frac{\sin (a t-(k+1 / 2) \pi)}{(a t-(k+1 / 2) \pi)},
\end{aligned}
$$


which reduces to (2.1) when $t=0, a=1$, and $\nu=0$. It also reduces to (2.2) when $t=0, a=1$, and $\nu$ is replaced by $\nu-\frac{1}{2}$. In both cases observe that $\sum_{k=-\infty}^{\infty}=2 \sum_{k=0}^{\infty}$.

One interesting consequence of (2.2) is the following formula which is obtained from (2.2) by taking the limit of both sides as $b \rightarrow 0$

$$
\left(\frac{\pi}{4}\right)^{\nu+1} \frac{1}{\Gamma(\nu+1)}=\sum_{k=0}^{\infty} \frac{(-1)^{k} J_{\nu}[\pi(k+1 / 2)]}{(2 k+1)^{\nu+1}}
$$

which, in turn, gives for $\nu=\frac{1}{2}$ the well-known formula $\sum_{k=0}^{\infty} 1 /(2 k+1)^{2}=$ $\pi^{2} / 8$.

Now we can employ the above argument to produce summation formulae, which we believe to be new.

In view of formula 6.739 in [8, p.762], we have

$$
\begin{aligned}
& \int_{0}^{\pi} J_{2 \nu}\left(b \sqrt{\pi^{2}-x^{2}}\right)\left(\pi^{2}-x^{2}\right)^{-1 / 2} \cos (t x) d x \\
& \quad=\frac{\pi}{2} J_{\nu}\left[\frac{\pi}{2}\left(\sqrt{b^{2}+t^{2}}+t\right)\right] J_{\nu}\left[\frac{\pi}{2}\left(\sqrt{b^{2}+t^{2}}-t\right)\right] .
\end{aligned}
$$

Thus, by putting $g(x, b)=J_{2 \nu}\left(b \sqrt{\pi^{2}-x^{2}}\right)\left(\pi^{2}-x^{2}\right)^{-1 / 2}$, which is in $L^{2}(0, \pi)$ for $\nu>\frac{1}{4}$, in (2.3), formulae (2.4) and (2.5), after some simple computations, become

$$
\begin{aligned}
J_{\nu}\left[\frac{\pi}{2}\left(\sqrt{b^{2}+t^{2}}+t\right)\right] J_{\nu}\left[\frac{\pi}{2}\left(\sqrt{b^{2}+t^{2}}-t\right)\right] \\
=\sum_{k=-\infty}^{\infty} J_{\nu}\left[\frac{\pi}{2}\left(\sqrt{b^{2}+\left(k+\frac{1}{2}\right)^{2}}+\left(k+\frac{1}{2}\right)\right)\right] \\
\times J_{\nu}\left[\frac{\pi}{2}\left(\sqrt{b^{2}+\left(k+\frac{1}{2}\right)^{2}}-\left(k+\frac{1}{2}\right)\right)\right] \frac{\sin \pi(t-(k+1 / 2))}{\pi(t-(k+1 / 2))},
\end{aligned}
$$

and

$$
\begin{aligned}
{\left[J_{\nu}\left(\frac{\pi b}{2}\right)\right]^{2}=2 \sum_{k=0}^{\infty} J_{\nu} } & {\left[\frac{\pi}{2}\left(\sqrt{b^{2}+\left(k+\frac{1}{2}\right)^{2}}+\left(k+\frac{1}{2}\right)\right)\right] } \\
\times J_{\nu} & {\left[\frac{\pi}{2}\left(\sqrt{b^{2}+\left(k+\frac{1}{2}\right)^{2}}-\left(k+\frac{1}{2}\right)\right)\right] \frac{(-1)^{k}}{\pi(k+1 / 2)} . }
\end{aligned}
$$

Upon replacing $b$ by $2 b$ in the last equation, we obtain for $\nu=\frac{1}{2}$

$$
\begin{aligned}
{[\sin \pi b]^{2}=2 \sum_{k=0}^{\infty} } & \sin \left[\pi\left(\sqrt{b^{2}+\left(\frac{k}{2}+\frac{1}{4}\right)^{2}}+\left(\frac{k}{2}+\frac{1}{4}\right)\right)\right] \\
& \times \sin \left[\pi\left(\sqrt{b^{2}+\left(\frac{k}{2}+\frac{1}{4}\right)^{2}}-\left(\frac{k}{2}+\frac{1}{4}\right)\right)\right] \frac{(-1)^{k}}{\pi(k+1 / 2)},
\end{aligned}
$$


which, in turn, gives as a special case for $b=\frac{1}{2}$

$$
\begin{aligned}
\frac{\pi}{4}=\sum_{k=0}^{\infty} & \sin \left[\pi\left(\sqrt{\frac{1}{4}+\left(\frac{k}{2}+\frac{1}{4}\right)^{2}}+\left(\frac{k}{2}+\frac{1}{4}\right)\right)\right] \\
& \times \sin \left[\pi\left(\sqrt{\frac{1}{4}+\left(\frac{k}{2}+\frac{1}{4}\right)^{2}}-\left(\frac{k}{2}+\frac{1}{4}\right)\right)\right] \frac{(-1)^{k}}{(2 k+1)} .
\end{aligned}
$$

We also obtain with the use of the identity $2 \sin A \sin B=\cos (A-B)-$ $\cos (A+B)$ that

$$
1-\cos 2 \pi b=\sum_{k=0}^{\infty} \cos \left[\pi \sqrt{4 b^{2}+\left(k+\frac{1}{2}\right)^{2}}\right] \frac{(-1)^{k+1}}{\pi(k / 2+1 / 4)},
$$

which gives for $b=1, \frac{1}{4}$

$$
\sum_{k=0}^{\infty} \cos \left[\pi \sqrt{4+\left(k+\frac{1}{2}\right)^{2}}\right] \frac{(-1)^{k+1}}{(2 k+1)}=0
$$

and

$$
\frac{\pi}{4}=\sum_{k=0}^{\infty} \cos \left[\pi \sqrt{\frac{1}{4}+\left(k+\frac{1}{2}\right)^{2}}\right] \frac{(-1)^{k+1}}{(2 k+1)} .
$$

By differentiating (2.6) with respect to $b$, we obtain (2.1) once more.

Another summation formula involving the Bessel functions, which we believe to be new, can be obtained as follows: From formula $6.681-1$ in [8, p. 738] we obtain

$$
\int_{0}^{\pi} J_{2 \nu}\left(2 b \cos \frac{x}{2}\right) \cos (t x) d x=\pi J_{\nu+t}(b) J_{\nu-t}(b) .
$$

Hence, by putting $g(x)=J_{2 \nu}\left(2 b \cos \frac{x}{2}\right)$, which is in $L^{2}(0, \pi)$ for $\nu>-\frac{1}{4}$, in (2.3), we then have $f(b, t)=\pi J_{\nu+t}(b) J_{\nu-t}(b)$, and consequently (2.4), (2.5) yield

$$
J_{\nu+t}(b) J_{\nu-t}(b)=\sum_{k=-\infty}^{\infty} J_{\nu+(k+1 / 2)}(b) J_{\nu-(k+1 / 2)}(b) \frac{\sin \pi(t-(k+1 / 2))}{\pi(t-(k+1 / 2))}
$$

and

$$
\left[J_{\nu}(b)\right]^{2}=\frac{4}{\pi} \sum_{k=0}^{\infty} J_{\nu+(k+1 / 2)}(b) J_{\nu-(k+1 / 2)}(b) \frac{(-1)^{k}}{(2 k+1)} .
$$

Similar summation formulae involving the modified Bessel functions of the first and second kinds $\mathbf{I}_{\nu}(b)$ and $\mathbf{K}_{\nu}(b)$, respectively, can also be obtained since they have integral representations similar to the one given in (2.7).

As a special case of (2.9), we obtain for $\nu=\frac{1}{2}$ that

$$
[\sin b]^{2}=\sum_{k=0}^{\infty} b J_{k+1}(b) J_{k}(b) \frac{2}{2 k+1} .
$$


Summation formulae involving other types of special functions can also be obtained as the next example shows.

From formula 6.685 in $[8$, p. 741$]$ we have

$$
\int_{0}^{\pi}\left(\sec \frac{x}{2}\right) \mathbf{K}_{2 \nu}\left(b \sec \frac{x}{2}\right) \cos (t x) d x=\frac{\pi}{b} \mathbf{W}_{t, \nu}(b) \mathbf{W}_{-t, \nu}(b),
$$

where $\mathbf{W}_{\lambda, \mu}(z)$ is the Whittaker function, which is related to the confluent hypergeometric function $\Psi(a, c ; z)$ by

$\mathbf{W}_{\lambda, \mu}(z)=e^{-z / 2} z^{c / 2} \Psi(a, c ; z), \quad$ where $\lambda=-a+c / 2$ and $\mu=(c-1) / 2$.

By putting $g(b, x)=\left(\sec \frac{x}{2}\right) \mathbf{K}_{2 \nu}\left(b \sec \frac{x}{2}\right)$ in (2.3), we obtain that $f(b, t)=$ $(\pi / b) \mathbf{W}_{t, \nu}(b) \mathbf{W}_{-t, \nu}(b)$; hence, formulae (2.4) and (2.5) take the form

$$
\mathbf{W}_{t, \nu}(b) \mathbf{W}_{-t, \nu}(b)=\sum_{k=-\infty}^{\infty} \mathbf{W}_{(k+1 / 2), \nu}(b) \mathbf{W}_{-(k+1 / 2), \nu}(b) \frac{\sin \pi(t-(k+1 / 2))}{\pi(t-(k+1 / 2))}
$$

and

$$
\left[\mathbf{W}_{o, \nu}(b)\right]^{2}=\frac{4}{\pi} \sum_{k=0}^{\infty} \mathbf{W}_{(k+1 / 2), \nu}(b) \mathbf{W}_{-(k+1 / 2), \nu}(b) \frac{(-1)^{k}}{(2 k+1)} .
$$

But since $\mathbf{W}_{o, \nu}(b)=\sqrt{b / \pi} \cdot \mathbf{K}_{\nu}(b / 2)$ (cf. [8, 9.235, p. 1062]), we then have

$$
b\left[\mathbf{K}_{\nu}\left(\frac{b}{2}\right)\right]^{2}=\frac{4}{\pi} \sum_{k=0}^{\infty} \mathbf{W}_{(k+1 / 2), \nu}(b) \mathbf{W}_{-(k+1 / 2), \nu}(b) \frac{(-1)^{2}}{(2 k+1)} .
$$

More formulae of similar types can be obtained in the same fashion.

\section{Summation formulae involving the Zeros of the Bessel function}

Now we turn our attention to a different type of summation formulae. These formulae, which involve the zeros of the Bessel function, will also be obtained from already known results in sampling theory.

The following result, which has been known for a while (cf. [16, Corollary 5, p. 904]), does not follow directly from the original version of Kramer's sampling theorem since the kernel of the integral transform $K(x, t)$ arises from a singular Sturm-Liouville problem. Nevertheless, it follows from Theorem 3.1 in [15] (cf. example 2-a in $§ 5.2$ ), where Kramer's sampling theorem has been extended to cases in which the kernel $K(x, t)$ arises from such a singular Sturm-Liouville problem.

If

$$
f(t)=\int_{0}^{1} g(x) \sqrt{x} J_{\nu}(t x) d x \text { for some } g \in L^{2}(0,1),
$$

then

$$
f(t)=\sum_{k=1}^{\infty} f\left(t_{k, \nu}\right) \frac{2 t_{k, \nu} J_{\nu}(t)}{J_{\nu+1}\left(t_{k, \nu}\right)\left[\left(t_{k, \nu}\right)^{2}-t^{2}\right]},
$$

where $t_{k, \nu}$ is the $k$ th positive zero of $J_{\nu}(t)$.

In view of formula $6.59-16$ in $[8$, p. 703$]$ we have

$$
\int_{0}^{1} x^{1 / 2-\nu}\left(1-x^{2}\right)^{\mu-1} \sqrt{x} J_{\nu}(t x) d x=\frac{2^{1-\nu} t^{-\mu}}{\Gamma(\nu)} \mathbf{s}_{\mu+\nu-1, \mu-\nu}(t), \quad \operatorname{Re} \mu>0,
$$


where $\mathbf{s}_{\mu, \nu}(z)$ is the Lommel function, which is defined in terms of the hypergeometric function by

$$
\mathbf{s}_{\mu, \nu}(z)=\frac{z^{\mu+1}}{(\mu-\nu+1)(\mu+\nu+1)}{ }_{1} \mathbf{F}_{2}\left(1 ; \frac{\mu-\nu+3}{2}, \frac{\mu+\nu+3}{2} ;-\frac{z^{2}}{4}\right),
$$

where $\mu \pm \nu$ is not a negative odd integer (cf. [8, 8574-3, p. 985]).

If we set $g(x)=x^{1 / 2-\nu}\left(1-x^{2}\right)^{\mu-1}$, which is in $L^{2}(0,1)$ for $\nu<1$ and $\mu>\frac{1}{2}$, in (3.1), we then have $f(t)=\left(2^{1-\nu} t^{-\mu} / \Gamma(\nu)\right) \mathbf{s}_{\mu+\nu-1, \mu-\nu}(t)$. Thus (3.2) now yields

$$
t^{-\mu} \mathbf{s}_{\mu+\nu-1, \mu-\nu}(t)=\sum_{k=1}^{\infty} \frac{2\left(t_{k, \nu}\right)^{1-\mu} \mathbf{s}_{\mu+\nu-1, \mu-\nu}\left(t_{k, \nu}\right) J_{\nu}(t)}{J_{\nu+1}\left(t_{k, \nu}\right)\left[\left(t_{k, \nu}\right)^{2}-t^{2}\right]} .
$$

But from (3.3), we have

$$
\mathbf{s}_{\mu+\nu-1, \mu-\nu}(t)=\frac{t^{\nu+\mu}}{4 \mu \nu}{ }_{1} \mathbf{F}_{2}\left(1 ; \nu+1, \mu+1 ; \frac{-t^{2}}{4}\right) .
$$

Hence, by taking the limit in (3.4) as $t \rightarrow 0$, we obtain after some simplifications

$$
2^{\nu-3} \frac{\Gamma(\nu)}{\mu}=\sum_{k=1}^{\infty} \frac{\mathbf{s}_{\mu+\nu-1, \mu-\nu}\left(t_{k, \nu}\right)}{J_{\nu+1}\left(t_{k, \nu}\right)\left(t_{k, \nu}\right)^{\mu+1}}
$$

which we believe is new.

In particular, if $\nu=\frac{1}{2}$, we have $t_{k, \nu}=k \pi$; hence (3.5) becomes

$$
\frac{\sqrt{\pi}}{\sqrt{2^{5}} \mu}=\sum_{k=1}^{\infty} \frac{\mathbf{s}_{\mu-1 / 2, \mu-1 / 2}(k \pi)}{J_{3 / 2}(k \pi)(k \pi)^{\mu+1}} .
$$

But since

$$
\mathbf{s}_{\nu, \nu}(\mathbf{z})=\Gamma\left(\nu+\frac{1}{2}\right) \sqrt{\pi} 2^{\nu-1} \mathbf{H}_{\nu}(\mathbf{z}) \quad(\text { cf. [8, 8.573-5, p. 986]), }
$$

where $\mathbf{H}_{\nu}(\mathbf{z})$ is the Struve function defined by

$$
\mathbf{H}_{\nu}(\mathbf{z})=\sum_{m=0}^{\infty}(-1)^{m} \frac{(\mathbf{z} / 2)^{2 m+\nu+1}}{\Gamma(m+3 / 2) \Gamma(\nu+m+3 / 2)} \quad \text { (cf. [8, 8.550-1, p. 982]), }
$$

equation (3.6) can now be written in the form

$$
\frac{1}{2^{\mu+1} \Gamma(\mu+1)}=\sum_{k=1}^{\infty} \frac{\mathbf{H}_{\mu-1 / 2}(k \pi)}{J_{3 / 2}(k \pi)(k \pi)^{\mu+1}} .
$$

It is easy to see that $J_{3 / 2}(k \pi)=\sqrt{2 / k} \cdot(-1)^{k+1} / \pi ;$ thus $(3.7)$ can be reduced to

$$
\frac{(\pi)^{\mu}}{2^{\mu+1 / 2} \Gamma(\mu+1)}=\sum_{k=1}^{\infty} \frac{(-1)^{k+1} \mathbf{H}_{\mu-1 / 2}(k \pi)}{(k)^{\mu+1 / 2}}
$$

see formula $(45)$ in $[3$, p. 70$]$; the series in $(3.8)$ is called a Schlömilch-type series. 
In view of the fact that $\mathbf{H}_{1 / 2}(z)=\sqrt{2 / \pi z} \cdot(1-\cos z)$ (cf. [8, 8,552-6, p. 983]), we obtain, from equality (3.8) for $\mu=1$, the well-known formula $\sum_{k=1}^{\infty} 1 /(2 k-1)=\pi^{2} / 8$.

Now let us start with Sonine's second integral $[3, \S 7.7,(4)]$

$$
\begin{gathered}
\int_{0}^{1} x^{\mu+1}\left(1-x^{2}\right)^{\nu / 2} J_{\nu}\left(b \sqrt{1-x^{2}}\right) J_{\mu}(t x) d x \\
=\frac{t^{\mu} b^{\nu}}{\left(t^{2}+b^{2}\right)^{(\mu+\nu+1) / 2}} J_{\nu+\mu+1}\left(\sqrt{t^{2}+b^{2}}\right),
\end{gathered}
$$

$t, b>0 ; \operatorname{Re} \nu, \operatorname{Re} \mu>-1$.

By setting $g(b, x)=x^{\mu+1 / 2}\left(1-x^{2}\right)^{\nu / 2} J_{\nu}\left(b \sqrt{1-x^{2}}\right)$ in (3.1), we then have

$$
f(b, t)=\frac{t^{\mu} b^{\nu}}{\left(t^{2}+b^{2}\right)^{(\nu+\mu+1) / 2}} J_{\nu+\mu+1}\left(\sqrt{t^{2}+b^{2}}\right),
$$

and consequently (3.2) gives

$$
\begin{aligned}
& \frac{t^{\mu}}{2 J_{\mu}(t)\left(t^{2}+b^{2}\right)^{(\nu+\mu+1) / 2}} J_{\nu+\mu+1}\left(\sqrt{t^{2}+b^{2}}\right) \\
& \quad=\sum_{k=1}^{\infty} \frac{\left(t_{k, \mu}\right)^{1+\mu}}{J_{\mu+1}\left(t_{k, \mu}\right)\left[\left(t_{k, \mu}\right)^{2}-t^{2}\right]} \frac{J_{\nu+\mu+1}\left(\sqrt{\left(t_{k, \mu}\right)^{2}+b^{2}}\right)}{\left[\left(t_{k, \mu}\right)^{2}+b^{2}\right]^{(\nu+\mu+1) / 2}},
\end{aligned}
$$

which is formula (4.1) in [7].

By taking the limit in the above equation as $t \rightarrow 0$, we obtain

$$
\frac{2^{\nu-1} \Gamma(\nu+1)}{b^{\nu+\mu+1}} J_{\nu+\mu+1}(b)=\sum_{k=1}^{\infty} \frac{\left(t_{k, \mu}\right)^{\mu-1}}{J_{\mu+1}\left(t_{k, \mu}\right)} \frac{J_{\nu+\mu+1}\left(\sqrt{\left(t_{k, \mu}\right)^{2}+b^{2}}\right)}{\left[\left(t_{k, \mu}\right)^{2}+b^{2}\right]^{(\mu+\nu+1) / 2}},
$$

from which upon taking the limit once more as $b \rightarrow 0$ we obtain

$$
\frac{\Gamma(\nu+1)}{2^{\mu+2} \Gamma(\nu+\mu+2)}=\sum_{k=1}^{\infty} \frac{J_{\nu+\mu+1}\left(t_{k, \mu}\right)}{J_{\mu+1}\left(t_{k, \mu}\right)\left(t_{k, \mu}\right)^{\nu+2}} .
$$

By taking the limit in (3.9) as $b \rightarrow 0$ we obtain

$$
\frac{J_{\nu+\mu+1}(t)}{2 J_{\mu}(t) t^{\nu+1}}=\sum_{k=1}^{\infty} \frac{J_{\nu+\mu+1}\left(t_{k, \mu}\right)}{J_{\mu+1}\left(t_{k, \mu}\right)\left(t_{k, \mu}\right)^{\nu}\left[\left(t_{k, \mu}\right)^{2}-t^{2}\right]},
$$

which can also be obtained from (3.1), (3.2), and Sonine's first integral (cf. [3, (5)]).

\section{SUMMATION FORMULAE INVOLVING THE $\Gamma$ FUNCTION}

In $\S 5.3$, example 4 of [15], the following sampling formula has been proved: if

$$
\begin{array}{r}
f(t)=\int_{0}^{\pi} g(\theta)\left(\sin \frac{\theta}{2}\right)^{\alpha+1 / 2}\left(\cos \frac{\theta}{2}\right)^{\beta+1 / 2} \mathbf{R}_{t-\gamma}^{(\alpha, \beta)}(\cos \theta) d \theta, \\
\alpha, \beta>-1,2 \gamma=\alpha+\beta+1,
\end{array}
$$


then

(4.2)

$$
f(t)= \begin{cases}\sum_{k=0}^{\infty} f(k+\gamma) \frac{(-1)^{k+1} 2(k+\gamma) \Gamma(k+2 \gamma)}{\Gamma(\gamma+t) \Gamma(\gamma-t)\left[t^{2}-(k+\gamma)^{2}\right] \Gamma(k+1)} & \text { if } \gamma \neq 0, \\ f(0) \frac{\sin \pi t}{\pi t}+\sum_{k=1}^{\infty} f(k) \frac{2 t \sin [\pi(t-k)]}{\pi\left[t^{2}-k^{2}\right]} & \text { if } \gamma=0,\end{cases}
$$

where $\mathbf{R}_{t}^{(\alpha, \beta)}(z)={ }_{2} \mathbf{F}_{1}(-t, t+2 \gamma ; \alpha+1 ;(1-z) / 2)$ is the Jacobi function, which reduces to the Jacobi polynomial of degree $n$ when $t=n$.

By substituting $x=\sin ^{2} \theta / 2$ in (4.1), we obtain

$$
f(t)=\int_{0}^{1} \hat{g}(x) x^{(\alpha / 2-1 / 4)}(1-x)^{(\beta / 2-1 / 4)}{ }_{2} \mathbf{F}_{1}(-t+\gamma, t+\gamma ; \alpha+1 ; x) d x
$$

where $\hat{g}(x)=g\left(2 \sin ^{-1} \sqrt{x}\right)$.

Also, by using the notation $(a)_{k}=\frac{\Gamma(a+k)}{\Gamma(a)}$ and the relations

$$
(x+k)=x(x+1)_{k} /(x)_{k}, \quad(x-k)=-\Gamma(1-x)(1-x)_{k} / \Gamma(-x)(-x)_{k},
$$

and $\Gamma(z) \Gamma(1-z)=\pi \csc (\pi z)$, we can transform (4.2) into the form

$$
f(t)=\sum_{k=0}^{\infty} f(k+\gamma) A_{k}(t, \gamma)
$$

where

$$
A_{k}(t, \gamma)=\frac{(-1)^{k} \Gamma(2 \gamma+1)(2 \gamma)_{k}(\gamma+1)_{k}(\gamma-t)_{k}(\gamma+t)_{k}}{\Gamma(\gamma+1+t) \Gamma(\gamma+1-t)(\gamma)_{k}(\gamma+1+t)_{k}(\gamma+1-t)_{k} \Gamma(k+1)},
$$

for $\gamma \neq 0$. A similar expression can be obtained for $\gamma=0$.

In view of formula $7.512-4[8, p .849]$ we have

$$
\int_{0}^{1} x^{\alpha}(1-x)^{\delta-1}{ }_{2} \mathbf{F}_{1}(-t+\gamma, t+\gamma ; \alpha+1 ; x) d x=\frac{\Gamma(\alpha+1) \Gamma(\delta) \Gamma(\delta-\beta)}{\Gamma(h+t) \Gamma(h-t)},
$$

where $h=(\alpha-\beta+1) / 2+\delta$ and $2 \gamma=\alpha+\beta+1$.

This last integral is in the form $(4.3)$ for $\hat{g}(x)=(x)^{(2 \alpha+1) / 4}(1-x)^{(4 \delta-2 \beta-3) / 4}$, which is in $L^{2}(0,1)$ for $\alpha>-1$ and where $\delta>(2 \beta+1) / 4$. Therefore, by substituting $f(t)=\Gamma(\alpha+1) \Gamma(\delta) \Gamma(\delta-\beta) / \Gamma(h+t) \Gamma(h-t)$ in (4.4), we obtain after some computations that for $\gamma \neq 0$

$$
\begin{aligned}
& \frac{\Gamma(h+\gamma) \Gamma(h-\gamma) \Gamma(1-t+\gamma) \Gamma(1+t+\gamma) \Gamma(\gamma)}{\Gamma(h+t) \Gamma(h-t) \Gamma(2 \gamma) \Gamma(\gamma+1)} \\
& =\sum_{k=0}^{\infty} \frac{2(\gamma-t)_{k}(\gamma+t)_{k}(\gamma+1)_{k}(2 \gamma)_{k}(1-h+\gamma)_{k}}{(1-t+\gamma)_{k}(1+t+\gamma)_{k}(\gamma)_{k}(h+\gamma)_{k} \Gamma(k+1)} \\
& =2{ }_{5} \mathbf{F}_{4}\left(\begin{array}{l}
2 \gamma, 1+\gamma, \gamma-t, \gamma+t, 1-h+\gamma ; \\
\gamma, 1+\gamma+t, 1+\gamma-t, h+\gamma ;
\end{array}\right),
\end{aligned}
$$

which is formula (III.12) in [11, p. 244] for $a=2 \gamma, b=\gamma-t, c=\gamma+t$, $d=1-h+\gamma$. 
Finally, we conclude this section with a summation formula involving the hypergeometric function ${ }_{3} \mathbf{F}_{2}$. This summation formula gives an expansion of a hypergeometric function in terms of hypergeometric polynomials.

In view of formula $7.51-5$ in $[8$, p. 849$]$ we have

$$
\begin{aligned}
\int_{0}^{1} x^{\delta-1}(1-x)^{\sigma-1}{ }_{2} \mathbf{F}_{1}(-t+\gamma, t+\gamma ; \alpha+1 ; x) d x \\
\quad=\frac{\Gamma(\delta) \Gamma(\sigma)}{\Gamma(\delta+\sigma)}{ }_{3} \mathbf{F}_{2}(-t+\gamma, t+\gamma, \delta ; \alpha+1, \delta+\sigma ; 1),
\end{aligned}
$$

$\operatorname{Re} \delta>0, \operatorname{Re} \sigma>\max \{0, \beta\}$.

This last integral is in the form (4.3) for $\hat{g}(x)=x^{(4 \delta-2 \alpha-3) / 4}(1-x)^{(4 \sigma-2 \beta-3) / 4}$, which is in $L^{2}(0,1)$ for $\delta>(2 \alpha+1) / 4$ and $\sigma>(2 \beta+1) / 4$.

Now with

$$
f(t)=\frac{\Gamma(\delta) \Gamma(\sigma)}{\Gamma(\delta+\sigma)}{ }_{3} \mathbf{F}_{2}(-t+\gamma, t+\gamma, \delta ; \alpha+1, \delta+\sigma ; 1),
$$

we obtain from (4.4) the summation formula

$$
\begin{aligned}
{ }_{3} \mathbf{F}_{2}(-t+\gamma, t+\gamma, \delta ; \alpha+1, \delta+\sigma ; 1) \\
\quad=\sum_{k=0}^{\infty}{ }_{3} \mathbf{F}_{2}(-k, k+2 \gamma, \delta ; \alpha+1, \delta+\sigma ; 1) A_{k}(t, \gamma),
\end{aligned}
$$

which, upon using the relation

$$
{ }_{2} \mathbf{F}_{1}(a, b ; c ; 1)=\frac{\Gamma(c) \Gamma(c-b-a)}{\Gamma(c-a) \Gamma(c-b)},
$$

and carrying out some computations, can be transformed once more, for $b_{1}=$ $\alpha+1$ and $b_{2}=\delta+\sigma$, into

$$
\begin{aligned}
& { }_{3} \mathbf{F}_{2}(-t+\gamma, t+\gamma, \delta ; \alpha+1, \delta+\sigma ; 1) \\
& =\sum_{k=0}^{\infty} \frac{(-1)^{k}(\gamma+t)_{k}(\gamma-t)_{k}}{(2 \gamma+k)_{k} \Gamma(k+1)} \mathbf{F}_{1}(k-t+\gamma, k+t+\gamma ; 1+2 k+2 \gamma ; 1) \\
& \quad \times{ }_{3} \mathbf{F}_{2}(-k, k+2 \gamma, \delta ; \alpha+1, \delta+\sigma ; 1) \\
& =\sum_{k=0}^{\infty} \frac{(-1)^{k}(\gamma+t)_{k}(\gamma-t)_{k}\left(b_{1}\right)_{k}\left(b_{2}\right)_{k}}{(2 \gamma+k)_{k} \Gamma(k+1)\left(b_{1}\right)_{k}\left(b_{2}\right)_{k}} \\
& \quad \times{ }_{4} \mathbf{F}_{3}\left(\begin{array}{l}
k+b_{1}, k+b_{2}, k+\gamma-t, k+\gamma+t ; \\
k+b_{1}, k+b_{2}, 1+2 k+2 \gamma ;
\end{array}\right) \\
& \quad \times{ }_{3} \mathbf{F}_{2}\left(\begin{array}{l}
-k, k+2 \gamma, \delta ; \\
b_{1}, b_{2} ;
\end{array}\right) .
\end{aligned}
$$

Formula (4.6) is a special case of formula (1.2) in [5] for $r=1, s=0$, $p=2=q, c_{1}=\delta, b_{1}=\alpha+1, b_{2}=\delta+\sigma, a_{1}=\gamma-t, a_{2}=\gamma+t, \beta=\delta+\sigma$, and $\alpha$ replaced by $\alpha+1, \gamma$ replaced by $2 \gamma$; see also [6]. 


\section{Summation formula involving the Kampé de FÉriet function}

In this section we derive a summation formula that is analogous to (4.5), but involving the Kampé de Fériet function. We will use the following contracted notation for the generalized hypergeometric function

$$
{ }_{p} F_{q}\left(\begin{array}{l}
a_{1}, \ldots, a_{p} ; \\
b_{1}, \ldots, b_{q} ;
\end{array}\right)={ }_{p} F_{q}\left(\begin{array}{l}
a_{P} ; \\
z \\
b_{Q} ;
\end{array}\right)={ }_{p} F_{q}\left(a_{P} ; b_{Q} ; z\right)=\sum_{k=0}^{\infty} \frac{\left(a_{P}\right)_{k} z^{k}}{\left(b_{Q}\right)_{k} k !},
$$

where $\left(a_{P}\right)_{k}=\prod_{j=1}^{p}\left(a_{j}\right)_{k},\left(b_{Q}\right)_{k}=\prod_{j=1}^{q}\left(b_{j}\right)_{k}$, and $(c)_{k}=\Gamma(c+k) / \Gamma(c)$.

Let $\hat{g}(x)=x^{\delta-\alpha / 2-3 / 4}(1-x)^{\sigma-\beta / 2-3 / 4}\left(\sum_{n=0}^{\infty} h_{n} x^{n}\right)$ be in $L^{2}(0,1)$ and assume that the series converges uniformly on $[0,1]$. Then by substituting this in (4.3), interchanging the summation and integration signs, and using formulae (2.3.2), (2.3.3) in [4], we obtain

$$
f(t)=\frac{\Gamma(\delta) \Gamma(\sigma)}{\Gamma(\delta+\sigma)} \sum_{n=0}^{\infty} h_{n} \frac{(\delta)_{n}}{(\delta+\sigma)_{n}}{ }_{3} F_{2}\left(\begin{array}{l}
-t+\gamma, t+\gamma, \delta+n ; \\
\alpha+1, \delta+\sigma+n
\end{array}\right) .
$$

Hence from (4.4) we have

$$
\begin{aligned}
& \sum_{n=0}^{\infty} h_{n} \frac{(\delta)_{n}}{(\delta+\sigma)_{n}}{ }_{3} F_{2}\left(\begin{array}{l}
-t+\gamma, t+\gamma, \delta+n ; \\
\alpha+1, \delta+\sigma+n ;
\end{array}\right) \\
& \quad=\sum_{k=0}^{\infty} \sum_{n=0}^{\infty} h_{n} \frac{(\delta)_{n}}{(\delta+\sigma)_{n}}{ }_{3} F_{2}\left(\begin{array}{l}
-k, k+2 \gamma, \delta+n ; \\
\alpha+1, \delta+\sigma+n ;
\end{array}\right) A_{k}(t, \gamma)
\end{aligned}
$$

As a special case of this, if we take $h_{n}=\left(a_{P}\right)_{n} /\left(b_{Q}\right)_{n} \cdot y^{n} / n$ ! with $p \leq q+1$, we obtain after some calculations that

$$
\begin{aligned}
F_{1: 1 ; q}^{1: 2 ; p} & \left(\begin{array}{l}
\delta:-t+\gamma, t+\gamma ;\left(a_{P}\right) ; \\
\delta+\sigma: \alpha+1 ;\left(b_{Q}\right) ;
\end{array}\right) \\
= & \sum_{k=0}^{\infty} F_{1: 1 ; q}^{1: 2 ; p}\left(\begin{array}{l}
\delta:-k, k+2 \gamma ;\left(a_{P}\right) ; \\
\delta+\sigma: \alpha+1 ;\left(b_{Q}\right) ;
\end{array}\right) A_{k}(t, \gamma),
\end{aligned}
$$

where

$$
\begin{aligned}
F_{I: m ; n}^{p: q ; k} & \left(\begin{array}{l}
\left(a_{P}\right):\left(b_{Q}\right) ;\left(c_{K}\right) ; \quad x, y \\
\left(\alpha_{L}\right):\left(\beta_{M}\right) ;\left(\gamma_{N}\right) ;
\end{array}\right) \\
= & \sum_{r, s=0}^{\infty} \frac{\prod_{i=1}^{p}\left(a_{i}\right)_{r+s} \prod_{i=1}^{q}\left(b_{i}\right)_{r} \prod_{i=1}^{k}\left(c_{i}\right)_{s}}{\prod_{i=1}^{l}\left(\alpha_{i}\right)_{r+s} \prod_{i=1}^{m}\left(\beta_{i}\right)_{r} \prod_{i=1}^{n}\left(\gamma_{i}\right)_{s}} \frac{x^{r}}{r !} \frac{y^{s}}{s !}
\end{aligned}
$$

is the Kampé de Fériet function. To the best of the author's knowledge, formula (5.1) is new. 


\section{ACKNOWLEDGMENT}

The author wishes to express his gratitude to the referee for some helpful suggestions and to Professor Mourad Ismail for his constructive comments that enabled the author to improve the results of this paper; in particular, for bringing to the author's attention the connection between formulae (4.2) and (4.6).

\section{REFERENCES}

1. P. Butzer, A survey of the Whittaker-Shannon sampling theorem and some of its extensions, J. Math. Res. Exposition 3 (1983), 185-212.

2. A. Erdelyi, W. Magnus, F. Oberhettinger, and F. Tricomi, Tables of integral transforms, vol. 1, McGraw-Hill, New York, 1954.

3. __ Higher transcendental functions, vol. 2, McGraw-Hill, New York, 1953.

4. H. Exton, Handbook of hypergeometric integrals, Wiley, New York, 1978.

5. J. Fields and M. Ismail, Polynomial expansions, Math. Comp. 29 (1975), 894-902.

6. J. Fields and J. Wimp, Expansions of hypergeometric functions in hypergeometric polynomials, Math. Comp. 15 (1961), 390-395.

7. R. Gosper, M. Ismail, and R. Zhang, On some strange summation formulas of Gosper, Illinois J. Math. (to appear).

8. I. Gradshteyn and I. Ryzhik, Tables of integrals, series and products, Academic Press, New York, 1965.

9. J. Higgins, Five short stories about the cardinal series, Bull. Amer. Math. Soc. (N.S.) 12 (1985), 45-89.

10. H. Kramer, A generalized sampling theorem, J. Math. Phys. 38 (1959), 68-72.

11. L. Slater, Generalized hypergeometric functions, Cambridge Univ. Press, Cambridge, England, 1966.

12. P. Weiss, Sampling theorem associated with Sturm-Liouville systems, Bull. Amer. Math. Soc. 63 (1957), 242.

13. A. Zayed, Kramer's sampling theorem for multi-dimensional signals and its relationship with Lagrange-type interpolation, J. Multidimensional Systems and Signal Processing (to appear).

14. A. Zayed, M. El-Sayed, and H. Annaby, On Lagrange interpolations and Kramer's sampling theorem associated with self-adjoint boundary-value problems, J. Math. Anal. Appl. 158 (1991), 269-284.

15. A. Zayed, On Kramer's sampling theorem associated with general Sturm-Liouville problems and Lagrange interpolation, SIAM J. Appl. Math. 51 (1991), 575-604.

16. A. Zayed, G. Hinsen, and P. Butzer, On Lagrange interpolation and Kramer-type sampling theorems associated with Sturm-Liouville problems, SIAM J. Appl. Math. 50 (1990), 893909.

Department of Mathematics, University of Central Florida, Orlando, Florida 32816 E-mail address: FDZAYED@UCF1VM-Bitnet 